\title{
Location Estimation Accuracy in Wireless Sensor Networks
}

\author{
Neal Patwari* and Alfred O. Hero III \\ Department of Electrical Engineering and Computer Science \\ University of Michigan, Ann Arbor, MI 48109
}

\begin{abstract}
The peer-to-peer nature of a wireless sensor network presents the opportunity for accurate and lowconfiguration sensor location estimation. Range measurements are made between pairs of sensors, regardless of their a priori coordinate knowledge. This paper quantifies via the Cramér-Rao Bound (CRB) variance limits on location estimators which use measured timeof-arrival (TOA) or received signal strength (RSS). An extensive campaign measures TOA and RSS in a 44-device multipoint-to-multipoint indoor network for input into maximum-likelihood estimators (MLEs) of location. RMS location errors of 1.2 and $2.2 m$ are demonstrated using TOA and RSS, respectively.
\end{abstract}

\section{Introduction}

Sensor location estimation in wireless sensor networks is both a requirement and an opportunity. To be useful, sensor data must be accompanied by location. Location estimation must be enabled in a manner consistent with the low power, low cost and low configuration requirements of sensor networks. The low power and low cost requirements preclude including GPS in each device, and the low configuration requirement prevents installation of a dense network of base stations. A low transmit power device may only be able to communicate with its nearby neighbors. However, when all devices in the network measure range to their neighbors, and a small proportion of devices, which we call reference devices, have a priori information about their coordinates, we have the opportunity to enable accurate sensor location estimates. We call this relative location estimation since it uses range measurements predominantly between pairs of devices of which neither has absolute coordinate knowledge.

Distributed algorithms [1] [11] [13] are proposed to locate devices in such wireless sensor networks using parallel and iterative estimation algorithms. If a central processor can be deployed, convex optimization [2] can solve a set of geometric constraints, or MLEs

*N. Patwari was employed at Motorola Labs, Plantation FL, USA, during the measurement campaign presented here. can be employed, as reported for sensors that measure angle-of-arrival and TOA [5] or RSS only [8].

This paper focuses on the sensor location accuracy possible in networks of devices capable of peer-to-peer RSS or TOA measurements. The radio channel is notorious for its impairments [6] [3], thus accurate RSS or TOA measurements are by no means a given. The CRBs presented in this article provide an ability to determine if the location accuracy necessary for a particular application is possible using either RSS or TOA.

First, we state the location estimation problem and model assumptions in Section 1.1, and derive the CRB and MLEs for the RSS and TOA cases in Sections 2 and 3 . Then, we present an extensive measurement campaign in Section 4, which we use to verify the channel model assumptions and to test the TOA and RSS relative location MLEs.

\subsection{Estimation problem statement}

We assume a wireless sensor network of $M$ reference devices and $N-M$ devices with unknownlocation, which we call blindfolded devices. The relative location problem is the estimation of $\theta=$ $\left\{x_{1}, \ldots, x_{N-M}, y_{1}, \ldots, y_{N-M}\right\}$ given the known coordinates, $\left\{x_{N-M+1}, \ldots, x_{N}, y_{N-M+1}, \ldots, y_{N}\right\}$. In the TOA case, $T_{i, j}$ is the measured TOA between devices $i$ and $j$ in (s), and in the RSS case, $P_{i, j}$ is the measured received power between devices $i$ and $j$ in (mW). The set $H(k) \subset\{1, \ldots, N\}$ is the set of all devices with which device $k$ has measured a range. By symmetry, if $l \in H(k)$ then $k \in H(l)$, and clearly $k \notin H(k)$. If reciprocal measurements (from $i$ to $j$ and then from $j$ to $i$ ) are made, we assume that they have been averaged together and set to $T_{i, j}$. For simplicity we consider $T_{i, j}$ and $P_{i, j}$ to be upper triangular.

We assume that $T_{i, j}$ is Gaussian distributed,

$T_{i, j} \sim \mathcal{N}\left(d_{i, j} / c, \sigma_{T}^{2}\right), \quad d_{i, j}=\sqrt{\left(x_{i}-x_{j}\right)^{2}+\left(y_{i}-y_{j}\right)^{2}}$ where $c$ is the speed of light, and $\sigma_{T}^{2}$ is not a function of $d_{i, j}$. We assume that $P_{i, j}$ is log-normal, thus the random variable $P_{i, j}(\mathrm{dBm})=10 \log _{10} P_{i, j}$ is Gaussian,

$$
\begin{aligned}
P_{i, j}(\mathrm{dBm}) & \sim \mathcal{N}\left(\bar{P}_{i, j}(\mathrm{dBm}), \sigma_{d B}^{2}\right) \\
\bar{P}_{i j}(\mathrm{dBm}) & =P_{0}(\mathrm{dBm})-10 n \log _{10}\left(d_{i, j} / d_{0}\right)
\end{aligned}
$$


where $P_{i, j}$ is the power received at device $i$ transmitted by device $j, \bar{P}_{i, j}(\mathrm{dBm})$ is the mean power in $\mathrm{dBm}$, and $Z_{i, j}(\mathrm{~dB})$ is the shadowing gain (loss) which is Gaussian when expressed in $\mathrm{dB}$. The mean received power is a function of $P_{0}(\mathrm{dBm})$, the free-space received power in $\mathrm{dBm}$ at a reference distance $d_{0}$, the path loss exponent $n$, and the distance $d_{i, j}$. We assume that the model parameters $d_{0}$ and $n$ are known or are estimated for the environment of interest. For simplicity, we assume that the data $T_{i, j}$ (and $P_{i, j}$ ) are independent $\forall i, j$.

These model assumptions will be shown to be valid in Section 4.1, using the literature and the results of the measurement campaign. In the next sections, we first use these model assumptions to derive the CRB and MLE for both the RSS and TOA cases.

\section{CRB for coordinate estimation}

The CRB provides a lower bound on the covariance matrix of any unbiased estimator of $\theta$. The $C R B$ is the inverse of the Fisher information matrix, $\mathbf{F}=-E\left[\nabla_{\theta}\left(\nabla_{\theta} l(\theta)\right)^{T}\right]$, where $l(\theta)=\log f_{p j \theta}(\mathbf{P} \mid \theta)$ is the $\log$ of the joint density function conditional on $\theta$. Since $\theta$ is a concatenation of $x$ and $y$ vectors, $\mathbf{F}$ partitions in both the RSS and TOA cases,

$$
\mathbf{F}_{R S S}=\left[\begin{array}{ll}
\mathbf{F}_{R x x} & \mathbf{F}_{R x y} \\
\mathbf{F}_{R x y}^{T} & \mathbf{F}_{R y y}
\end{array}\right], \quad \mathbf{F}_{T O A}=\left[\begin{array}{ll}
\mathbf{F}_{T x x} & \mathbf{F}_{T x y} \\
\mathbf{F}_{T x y}^{T} & \mathbf{F}_{T y y}
\end{array}\right] .
$$

In the RSS case,

$$
\begin{gathered}
f_{p \mid \theta}(\mathbf{P} \mid \theta)=\prod_{i=1}^{N-1} \prod_{\substack{j \in H(i) \\
j<i}} \frac{10 / \log 10}{\sqrt{2 \pi \sigma_{d B}^{2}}} \frac{1}{P_{i, j}} e^{-\frac{b}{8}\left(\log \frac{d_{i, j}^{2}}{d_{i, j}^{2}}\right)^{2}} \\
\text { where } b=\left(\frac{10 n}{\sigma_{\mathrm{d} B} \log 10}\right)^{2}, \quad \tilde{d}_{i, j}=d_{0}\left(\frac{P_{0}}{P_{i, j}}\right)^{1 / n} .
\end{gathered}
$$

To see the physical meaning behind the measured power, consider that $\tilde{d}_{i, j}$ has units of $(\mathrm{m})$ and is actually the MLE of range $d_{i, j}$ given $P_{i, j}$. Thus,

$$
l(\theta)=\sum_{i=1}^{N-1} \sum_{\substack{j \in H(i) \\ j<i}}\left[C_{1}-\frac{b}{8}\left(\log \frac{d_{i, j}^{2}}{\tilde{d}_{i, j}^{2}}\right)^{2}\right]
$$

where $C_{1}$ is a term which is constant w.r.t. $\theta$. The $2^{\text {nd }}$ partial derivative of $(2)$ w.r.t. $\theta_{\mathrm{r}}$ and $\theta_{s}$ will be a summation of terms if $\theta_{r}$ and $\theta_{s}$ are coordinates of the same device $k$, but will be only one term if $\theta_{r}$ and $\theta_{s}$ are coordinates of different devices $k$ and $l, k \neq l$. For example,

$$
\begin{aligned}
& \frac{\partial^{2} l(\theta)}{\partial x_{k} \partial y_{k}}=-b \sum_{i \in H(k)} \frac{\left(x_{i}-x_{k}\right)\left(y_{i}-y_{k}\right)}{d_{i, k}^{4}}\left[-\log \frac{d_{i, k}^{2}}{d_{i, k}^{2}}+1\right] \\
& \frac{\partial^{2} l(\theta)}{\partial x_{k} \partial y_{l}}=-b \mathrm{I}_{H(k)}(l) \frac{\left(\mathrm{x}_{1}-x_{k}\right)\left(y_{1}-y_{k}\right)}{\mathrm{d}_{l, k}^{4}}\left[\log \frac{\mathrm{d}_{1, k}^{2}}{\mathrm{~d}_{1, k}^{2}}-1\right]
\end{aligned}
$$

where $\mathrm{I}_{H(\mathrm{k})}(1)=1$ if $l \in H(k)$ and 0 otherwise. All of the $2^{\text {nd }}$ partial derivatives depend on a term, $\log \left(d_{i, k}^{2} / \tilde{d}_{i, k}^{2}\right)$, which has an expected value of zero. The elements of $F_{R S S}$ become

$$
\begin{aligned}
& \left(\left(F_{R x x}\right)\right)_{k, l}= \begin{cases}b \sum_{i \in H(k)} \frac{\left(x_{k}-x_{i}\right)^{2}}{\left[\left(x_{k}-x_{i}\right)^{2}+\left(y_{k}-y_{i}\right)^{2}\right]^{2}} & k=l \\
-b \mathrm{I}_{\mathrm{H}(\mathrm{k})}(1) \frac{\left(\mathrm{x}_{\mathrm{k}}-\mathrm{x}_{1}\right)^{2}}{\left.\left(\mathrm{x}_{\mathrm{k}}-\mathrm{x}_{1}\right)^{2}+\left(\mathrm{y}_{\mathrm{k}}-\mathrm{y}_{1}\right)^{2}\right]^{2}} & k \neq l\end{cases} \\
& \left(\left(F_{R x y}\right)\right)_{k, l}= \begin{cases}b \sum_{i \in H(k)} \frac{\left(x_{k}-x_{i}\right)\left(y_{k}-y_{i}\right)}{\left.\left(x_{k}-x_{i}\right)^{2}+\left(y_{k}-y_{i}\right)^{2}\right)^{2}} & k=l \\
-b \mathrm{I}_{\mathrm{H}(\mathrm{k})}(1) \frac{\left(x_{k}-x_{1}\right)\left(y_{k}-y_{i}\right)}{\left(\frac{\left.\left.x_{k}-x_{1}\right)^{2}+\left(y_{k}-y_{1}\right)^{2}\right)^{2}}{2}\right.} & k \neq l\end{cases} \\
& \left(\left(F_{R y y}\right)\right)_{k, l}=\left\{\begin{array}{cc}
b \sum_{i \in H(k)} \frac{\left(y_{k}-y_{i}\right)^{2}}{\left[\left(x_{k}-x_{i}\right)^{2}+\left(y_{k}-y_{i}\right)^{2}\right]^{2}} & k=l \\
-b \mathrm{I}_{\mathrm{H}(\mathrm{k})}(1) \frac{\left(\mathrm{y}_{\mathrm{k}}-\mathrm{y}_{j}\right)^{2}}{\left[\left(\mathrm{x}_{\mathrm{k}}-\mathrm{x}_{1}\right)^{2}+\left(\mathrm{y}_{\mathrm{k}}-\mathrm{y}_{1}\right)^{2}\right]^{2}} & k \neq l
\end{array}\right.
\end{aligned}
$$

For the TOA case, the derivation is very similar and is omitted for brevity. The elements of the submatrices of $\mathbf{F}_{\text {TOA }}$ are given by

$$
\begin{aligned}
& \left(\left(F_{T x x}\right)\right)_{k, l}= \begin{cases}\frac{1}{c^{2} \sigma_{T}^{2}} \sum_{i \in H(k) \frac{\left(x_{k}-x_{i}\right)^{2}}{\left(x_{k}-x_{i}\right)^{2}+\left(y_{k}-y_{i}\right)^{2}}} & k=l \\
-\frac{1}{c^{2} \sigma_{T}^{2}} \mathrm{I}_{\mathrm{H}(\mathrm{k})}(1) \frac{\left(x_{k}-x_{1}\right)^{2}}{\left(x_{\mathrm{k}}-x_{1}\right)^{2}+\left(y_{k}-y_{1}\right)^{2}} & k \neq l\end{cases} \\
& \left(\left(F_{T x y}\right)\right)_{k, l}= \begin{cases}\frac{1}{c^{2} \sigma_{T}^{2}} \sum_{i \in H(k)} \frac{\left(x_{k}-x_{i}\right)\left(y_{k}-y_{i}\right)}{\left(x_{k}-x_{i}\right)^{2}+\left(y_{k}-y_{i}\right)^{2}} & k=l \\
-\frac{1}{c^{2} \sigma_{T}^{2}} \mathrm{I}_{H(\mathrm{k})}(1) \frac{\left(x_{k}-x_{1}\right)\left(y_{k}-y_{1}\right)}{\left(x_{k}-x_{1}\right)^{2}+\left(y_{k}-y_{1}\right)^{2}} & k \neq l\end{cases} \\
& \left(\left(F_{T y y}\right)\right)_{k, l}= \begin{cases}\frac{1}{c^{2} \sigma_{T}^{2}} \sum_{i \in H(k)} \frac{\left(y_{k}-y_{i}\right)^{2}}{\left(x_{k}-x_{i}\right)^{2}+\left(y_{k}-y_{i}\right)^{2}} & k=l \\
-\frac{1}{c^{2} \sigma_{T}^{2}} \mathrm{I}_{\mathrm{H}(\mathrm{k})}(1) \frac{\left(y_{k}-y_{1}\right)^{2}}{\left(x_{k}-x_{l}\right)^{2}+\left(y_{k}-y_{i}\right)^{2}} & k \neq l\end{cases}
\end{aligned}
$$

Note $\mathbf{F}_{R S S}$ is proportional to $n / \sigma_{d B}$ while $\mathbf{F}_{T O A}$ is proportional to $1 /\left(c^{2} \sigma_{T}^{2}\right)$. These two signal-to-noise ratio quantities directly affect the $\mathrm{CRB}$. Also, in the TOA case, the dependence on the coordinates is in unitless distance ratios. These indicate that the size of the system can be scaled without changing the CRB as long as the geometry is kept the same. However, in the RSS case, due to the $d^{4}$ terms in the denominator of each term of $\mathbf{F}_{R S S}$ the variance bound must increase with to the size of the system even if the geometry is kept the same. These scaling characteristics indicate that TOA will be preferred for sparse networks, but at some high density, RSS can perform as well as TOA.

\subsection{Existing location system example}

Consider the simple case when device 1 is blindfolded and devices $2 \ldots N$ are references. This example is equivalent to many existing location systems in the literature, and a bound for the variance of the location estimator has been derived in the TOA case [12]. There are only two unknowns in this case, $x_{1}$ and $y_{1}$. The CRB for location estimators in this example we denote $\sigma_{1}^{2}$. In the RSS case,

$$
\begin{gathered}
E\left[\left(\hat{x}_{1}-x_{1}\right)^{2}+\left(\hat{y}_{1}-y_{1}\right)^{2}\right] \leq \sigma_{1}^{2}=\frac{F_{R x x}+F_{R y y}}{F_{R x x} F_{R y y}-F_{R x y}^{2}} \\
\sigma_{1}^{2}=\frac{1}{b} \frac{\sum_{i=2}^{N} \frac{d_{1, i}^{-2}}{\sum_{i=2}^{N-1} \sum_{j=i+1}^{N}\left(\frac{d_{1, i, j} d_{i, j}}{d_{1, i}^{2} d_{1, j}^{2}}\right)^{2}}}{}
\end{gathered}
$$



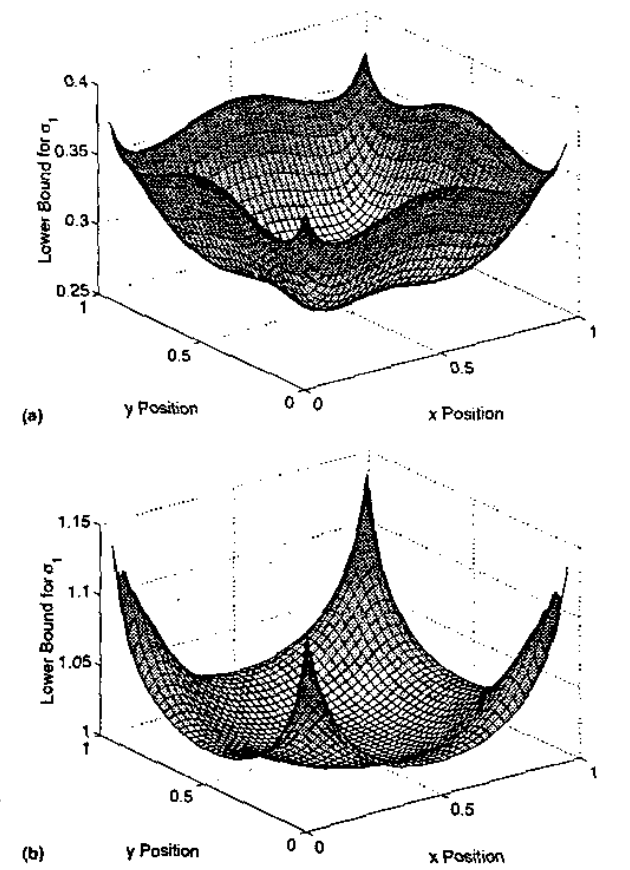

Figure 1: $\sigma_{1}(\mathrm{~m})$ for the example system vs. the coordinates of the single blindfolded device, for (a) RSS with $\sigma_{d B} / n=1.7$, or (b) TOA with $c \sigma_{T}=1$.

where the distance $d_{1 \perp i, j}$ is the shortest distance from the point $\left(x_{1}, y_{1}\right)$ to the line between device $i$ and device $j$. For the TOA case,

$$
\sigma_{1}^{2}=c^{2} \sigma_{T}^{2} m\left[\sum_{i=2}^{N-1} \sum_{j=i+1}^{N}\left(\frac{d_{1 \perp i, j} d_{i, j}}{d_{1, i} d_{1, j}}\right)^{2}\right]^{-1}
$$

The ratio $d_{1 \perp i, j} d_{i, j} /\left(d_{1, i} d_{1, j}\right)$ has been called the geometric conditioning $\mathcal{A}_{i, j}$ of device 1 with respect to references $i$ and $j[12] . \mathcal{A}_{i, j}$ is the area of the parallelo gram formed by the vectors from device 1 to reference $i$ and from device 1 to reference $j$, normalized by the lengths of the two vectors. Thus the geometric dilution of precision (GDOP), defined as $\sigma_{1} /\left(c \sigma_{T}\right)$, is

$$
G D O P=\sqrt{\frac{m}{\sum_{i=-m+1}^{-1} \sum_{j=i+1}^{0} \mathcal{A}_{i, j}^{2}}},
$$

which matches the result in [12]. The bound in (3) is constant with scale if $\mathcal{A}_{i, j}$ is unchanged $\forall i, j$.

Contour plots of $\sigma_{1}$ for the RSS and TOA cases are shown in Fig. 1 when there are four reference devices located in the corners of a $1 \mathrm{~m}$ by $1 \mathrm{~m}$ square. The minimum value in Fig. 1(a) is 0.27 . Since the CRB scales with size in the RSS case, the standard deviation of location estimates in a traditional RSS system with $\sigma_{d B} / n=1.7$ is limited to about $27 \%$ of the distance between reference devices. This performance has prevented use of RSS in many existing location systems and motivates the use of relative location information. In the TOA case in Fig. 1(b), $\sigma_{1} \propto c \sigma_{T}$, thus $c \sigma_{T}=1$ was chosen for ease of calculation.

\section{Relative location MLEs}

A maximum likelihood estimation algorithm is shown in [8] for the two-dimensional RSS case. Here, we consider a bias-reduced MLE for the RSS case,

$$
\hat{\theta}=\arg \min \sum_{i=1}^{N-1} \sum_{\substack{j \in H(i) \\ j<i}}\left(\ln \frac{\tilde{d}_{i, j}^{2}}{C^{2} d_{i, j}^{2}}\right)^{2}
$$

where $C=\exp \left[0.5\left(\sigma_{d B} \log 10\right)^{2} /(10 n)^{2}\right]$. To see the bias-reduction, consider the case when $M=1, N=2$. With only two devices, (4) will place the blindfolded device such that $d_{i, j}^{2}=\tilde{d}_{i, j}^{2} / C^{2}$. Since $E\left[\tilde{d}_{i, j}\right]=C d_{i, j}$, (4) makes the separation of the two devices unbiased.

The RSS bias-reduced MLE is still a biased estimator. For the example in Section 2.1 with $M=4$ and $N=5$, the bias is very high near the edges of the square area. Shown in Fig. 2 is the estimated bias gradient norm of $\hat{x}_{1}$, which can be used to find the uniform CRB [4]. Intuitively, (4) tries to force the ratio $\tilde{d}_{1, j}^{2} /\left(C^{2} d_{1, j}^{2}\right)$ close to 1 . When $\tilde{d}_{1, j}^{2}$ is small, the estimator has little freedom to place device 1 with respect to device $j$. In the limit as the actual locations of devices 1 and $j$ become equal, the MLE will locate device 1 at device $j$ with zero variance. It makes sense that the simulated bias gradient norm is close to 1 at the corners of Fig. 2.

For the TOA case, the MLE is given by

$$
\hat{\theta}=\arg \min \sum_{i=1}^{N-1} \sum_{j=i+1}^{N}\left(c T_{i, j}-d_{i, j}\right)^{2} .
$$

\section{Channel measurement experiment}

In this section, we describe the measurement system and experiment and show why the channel model assumptions made in Section 1.1 are valid. The channel measurements are conducted in the Motorola facility in Plantation, Florida in a $14 \mathrm{~m}$ by $13 \mathrm{~m}$ cubicle area. The cubicles have $1.8 \mathrm{~m}$ high walls and are occupied with desks, bookcases, metal and wooden filing cabinets, computers and equipment. There are also metal and concrete support beams within and outside of the area. Forty-four device locations are identified and marked with tape.

The measurement system uses a wideband directsequence spread-spectrum (DS-SS) transmitter (TX) 


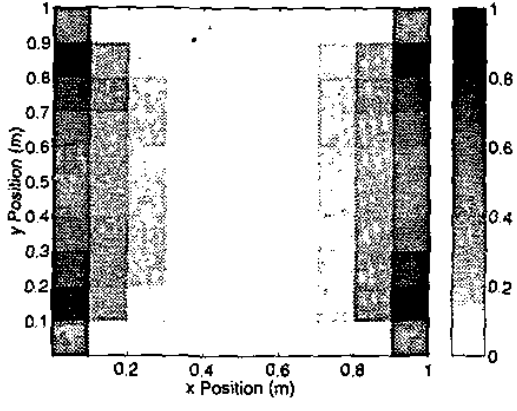

Eigure 2: Bias gradient norm of the RSS MLE of $x_{1}$ from (4) for the example system of Section 2.1.

and receiver (RX) (Sigtek ST-515). They are operated synchronously using two Datum ExacTime GPS $\&$ rubidium-based oscillators. The TX outputs an unmodulated pseudo-noise (PN) signal with a $40 \mathrm{MHz}$ chip rate, code length 1024 , center frequency $f_{c}$ of $2443 \mathrm{MHz}$, and TX power $P_{t}$ of $10 \mathrm{~mW}$. The RX takes complex samples at $120 \mathrm{MHz}$, downconverts, and correlates them with the known PN signal. Both TX and $\mathrm{RX}$ use $2.4 \mathrm{GHz}$ sleeve dipole antennas kept at a height of $1 \mathrm{~m}$ above the floor. The antennas have an omni pattern in the horizontal plane and a measured gain of $1.1 \mathrm{dBi}$. Periodic time calibrations are made to enable a time base accuracy of 1-2 ns, and power calibrations are done ensure accurate RSS measurement.

During the campaign, the channel between each pair of device locations is measured. First, the TX is placed at location 1 while the RX is moved to Jocations 2 through 44 . Then the TX is moved to location 2, as the RX is moved to locations 1 and 3 to 44. At each combination of TX and RX locations, the $R X$ records five wideband channel measurements. All devices are in range of all other devices, so a total of $44^{*} 43^{*} 5=9460$ wideband channels are measured. Since we expect reciprocity, each link has a total of 10 measurements that can be averaged.

\subsection{Estimating TOA and RSS}

The wideband radio channel is typically modeled as a sum of attenuated, phase-shifted, and time delayed multipath impulses [3] [10]. The power-delay profile (PDP) output of the Sigtek measurement system, due to its finite bandwidth, replaces each impulse of the channel impulse response with the autocorrelation function of the PN signal $R_{P N}(\tau)$, a triangular peak $2 / R_{C}$ wide. The line-of-sight (LOS) component, with TOA $d_{i, j} / c$, can be obscured by non-LOS multipath that arrive within $2 / R_{C}$ after the LOS TOA. If the LOS component is attenuated more than the early-arriving multipath, it can be difficult to distin- quish the LOS TOA.

We estimate the LOS TOA by template-matching [9], in which samples of the leading edge of the PDP are compared to an oversampled template of $R_{P N}(\tau)$. The TOA estimate $\bar{t}_{i, j}$ is the delay that minimizes the squared-error between the samples of the PDP and the template. Due to the fact that the non-LOS multipath are delayed in time, $\tilde{t}_{i, j}$ usually has a positive bias. We estimate the bias to be the average of $\tilde{t}_{i, j}-d_{i, j} / c, \forall i, j$ which in these measurements is $10.9 \mathrm{~ns}$. Subtracting out the bias, we get the unbiased TOA estimator $t_{i, j}$. Finally, the average of the $10 t_{i, j}$ measurements for the link between $i$ and $j$ we call $T_{i, j}$. The measured standard deviation, $\sigma_{T}$, is $6.1 \mathrm{~ns}$.

It has been shown that a wideband estimate of received power, $p_{i, j}$, is obtained by summing the powers of the multipath of the PDP [10]. This wideband method reduces the frequency-selective fading effects. The geometric mean of the $10 p_{i, j}$ measurements for the link between $i$ and $j$, called $P_{i, j}$, reduces fading due to motion of objects in the environment. Shadowing effects, caused by permanent obstructions in the channel, remain predominant in $P_{i, j}$ since sensors are assumed to be stationary. Shadowing loss is often reported to be a log-normal random variable [3][10], which leads to the log-normal shadowing model in (1). The measured $P_{i, j}$ match the log-normal shadowing model in (1) with $n=2.30$ and $\sigma_{d B}=3.92 \mathrm{~dB}$, using $d_{0}=1 \mathrm{~m}$. The low variance may be due both to the wide bandwidth and averaging, and to the homogeneity of the measured cubicle area.

$P_{i, j}$ and $T_{i, j}$ for the link $i-j$ is a random function not of time but of place. This is because the obstructions between devices $i$ and $j$ that cause shadowing and obstruction of the LOS don't change over time. However the two devices placed the same distance apart in a different area would have a different realization. Still, we can experimentally see the log-normal and Gaussian distributions of the RSS and TOA measurements if we examine $P_{i, j}(\mathrm{dBm})-\tilde{P}_{i, j}(\mathrm{dBm})$ and $T_{i, j}-d_{i, j} / c$. Both are demonstrated to have a very close fit to the Gaussian distribution using quantile-quantile plots [7].

\subsection{Experimental results}

The RSS and TOA measurements $P_{i, j}$ and $T_{i, j}$ are input to the MLEs in (4) and (5). The minimum in each case is found via a conjugate gradient algorithm. The estimated device locations are compared to the actual locations in Fig. 3(a) and (b). To generalize the results, the RMS location error of all 40 unknown-location devices is $2.18 \mathrm{~m}$ in the RSS case and $1.23 \mathrm{~m}$ in the TOA case. Since shadowing and non-LOS errors are not ergodic, calculating the MLE variances requires several measurement campaigns in 
different areas. This was not possible due to time limitations. But we note that the root mean variance bound, $\left(\sum_{j=1}^{40} \sigma_{i}^{2} / 40\right)^{1 / 2}$, is equal to $0.76 \mathrm{~m}$ for the RSS case and 0.69 in the TOA case. We also notice that the devices close to the center are located more accurately than the devices on the edges, particularly in the RSS case. Devices at the edges have fewer nearby neighbors to benefit their location estimate.

\section{Conclusion}

In a measured network in an office area, we show location errors in the RSS case about twice those observed in the TOA case. From the CRB results, we know that at some density, a location system can perform as well using RSS as TOA. Since RSS is a less costly feature to implement in hardware, the results are important to the development of low-cost wireless sensor networks. In general, the results in this paper should allow designers of wireless sensor networks to determine if the accuracy possible can meet their requirements. Future research may use the CRB to evaluate new coordinate estimators. Also, if a model of the joint distribution of TOA and RSS can be determined, then a CRB can be determined for estimators using both TOA and RSS data.

\section{Acknowledgments}

We would like to acknowledge the contribution of Miguel Roberts and Neiyer Correal, who assisted with the measurement system.

\section{References}

[1] J. Albowicz, A. Chen, and L. Zhang. Recursive position estimation in sensor networks. In IEEE Int. Conf. on Network Protocols, pp. 35-41, Nov 2001.

[2] L. Doherty, K. S. pister, and L. E. Ghaoui. Convex position estimation in wireless sensor networks. In IEEE INFOCOM, vol. 3, pp. $1655-1663,2001$.

[3] H. Hashemi. The indoor radio propagation channel. Proc. of the IEEE, 81(7):943-968, July 1993.

[4] A. O. Hero, J. A. Fessler, and M. Usman. Exploring estimator bias-variance tradeoffs using the uniform $\mathrm{cr}$ bound. IEEE Trans. on Sig. Proc., 44(8):2026-2041, Aug. 1996.

[5] R. L. Moses, D. Krishnamurthy, and R. Patterson. An auto-calibration method for unattended ground sensors: In ICASSP, vol. 3, pp. 2941-2944, May 2002.

[6] K. Pahlavan, P. Krishnamurthy, and J. Beneat. Wideband radio propagation modeling for indoor geolocation applications. IEEE Comm. Mag., pp. 60-65, April 1998

[7] N. Patwari, A. O. H. III, M. Perkins, N. S. Correal, and R. J. O'Dea. Relative location estimation in wireless sensor networks. submitted to IEEE Trans. on Sig. Proc.

[8] N. Patwari, R. J. O'Dea, and Y. Wang. Relative location in wireless networks. In IEEE VTC, vol, 2, pp. 1149-1153, May 2001.

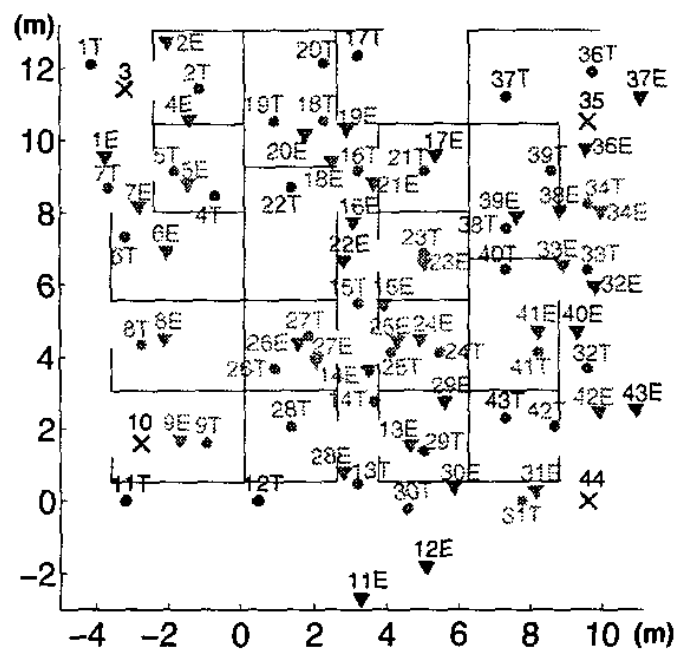

(a)

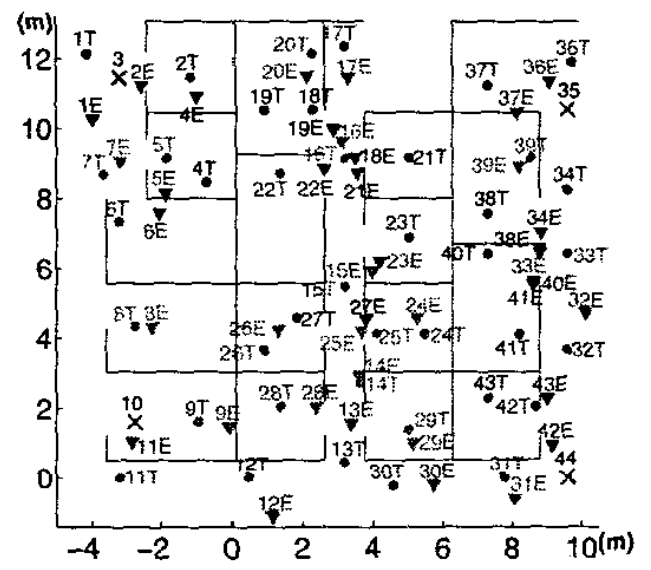

Figure 3: True $(\bullet \# T)$ and estimated $(\mathbf{\nabla}$ E) location using (a) RSS and (b) TOA data for measured network with 4 reference devices (X\#). Higher errors are indicated by darker text.

[9] B. B. Peterson, C. Kmiecik, R. Hartnett, P. M. Thompson, J. Mendoza, and H. Nguyen. Spread spectrum indoor geolocation. Journal of the Inst. of Navigation, 45(2):97-102, Summer 1998.

[10] T. Rappaport. Wireless Communications: Principles and Practice. Prentice-Hall Inc., New Jersey, 1996.

[11] C. Savarese, J. M. Rabaey, and J. Beutel. Locationing in distributed ad-hoc wireless sensor networks. In ICASSP, pp. 2037-2040, May 2001:

[12] M. A. Spirito. On the accuracy of cellular mobile station location estimation. IEEE Trans. on Veh. Tech., 50(3):674-685, May 2001.

[13] S. Capkun, M. Hamdi, and J.-P. Hubaux. GPS-free positioning in mobile ad-hoc network. In $34^{\text {th }}$ IEEE Hawaii Int. Conf. on System Sciences (HICSS-34), Jan. 2001. 